\title{
Migraine with Brainstem Aura Accompanied by Disorders of Consciousness
}

\author{
Sui-yi Xu* \\ Hui-juan Li* \\ Jing Huang \\ Xiu-ping Li \\ Chang-xin Li $\mathbb{D}$
}

Department of Neurology, The First Hospital of Shanxi Medical University, Taiyuan, 03000I, Shanxi Province,

People's Republic of China

*These authors contributed equally to this work
Correspondence: Chang-xin $\mathrm{Li}$ Department of Neurology, The First Hospital of Shanxi Medical University, Jiefangnan Road 85, Taiyuan, 03000I, Shanxi Province, People's Republic of China

Tel +86-15103513579

Email lichangxin035I@sina.com

\begin{abstract}
Migraine with brainstem aura (MBA) accompanied by disorders of consciousness (DOC) is a rare subtype of migraine. The pathophysiology of MBA with DOC has not been elucidated yet. Some patients have a family history of migraine, and women are more affected than men. The aura symptoms are diverse; however, when MBA is combined with DOC, the clinical manifestations are more complicated. Coma is the most common clinical manifestation. The overall duration of the patient's DOC is short and can often return to normal within half an hour. Headache often occurs after regaining consciousness and can also occur at the same time as DOC. The most common headache is located at the occipital region. Although DOC is reversible, considering the current small number of cases, we still need to improve our understanding of the disease to avoid misdiagnosis. The MBA patient's electroencephalogram and cerebral blood flow perfusion may have transient changes and may return to normal in the interictal period or after the DOC. Although triptans have traditionally been contraindicated in MBA under drug instructions, the evidence of basilar artery constriction, as postulated in MBA, is lacking. Lasmiditan is currently the first and only 5-HT 1F receptor agonist approved by the Food and Drug Administration. The calcitonin generelated peptide receptor antagonists and monoclonal antibody therapies may be the most promising for future consideration. Here, the pathophysiology, clinical manifestations, diagnostic tools, and treatment progress for MBA with DOC are reviewed.
\end{abstract}

Keywords: migraine, brainstem aura, coma, calcitonin gene-related peptide

\section{Introduction}

Migraine with brainstem aura (MBA) is a rare migraine subtype ${ }^{1-15}$ and accounts for about $1.5 \%$ of headache and $6.6-10 \%$ of migraine with aura. ${ }^{16,17}$ The aura features include vertigo, dysarthria, diplopia, tinnitus, ataxia, and disorders of consciousness (DOC). ${ }^{18}$ Hiccups $^{19}$ or exploding head syndrome (EHS) ${ }^{14}$ may also occur in some patients. In 1961, Edwin reported for the first time a subtype of migraine with brainstem dysfunction and proposed the concept of basilar artery migraine. ${ }^{20}$ Since then, impairment of consciousness in migraine was considered as a prominent aura symptom. ${ }^{1}$ At present, Headache Classification Committee of the International Headache Society (IHS) has renamed basilar artery migraine to $\mathrm{MBA} .{ }^{18}$ It is notable that the complex symptoms and signs of DOC can negatively affect the differential diagnosis for the DOC-accompanied primary disorder. ${ }^{21}$ Therefore, more attention should be paid to achieve early recognition, rapid diagnosis, and timely treatment.

\section{Pathophysiology of MBA with DOC}

The pathophysiology of MBA has not been elucidated yet. ${ }^{22}$ It mainly includes three hypotheses: vasomotor dysfunction, ${ }^{23}$ cortical spreading depression, ${ }^{24,25}$ and 
neurogenic inflammation. ${ }^{26-28}$ Regarding the MBA with DOC, abnormal neurotransmitter secretion may cause reticular activating system (RAS) dysfunction. ${ }^{5,10}$ Increased secretion of the inhibitory neurotransmitter $\gamma$-aminobutyric acid (GABA) in migraine patients ${ }^{29}$ may be involved in the dysfunction of RAS, thereby changing the state of consciousness. $^{30}$ The hypothalamus plays an important role in migraine. Resting regional cerebral blood flow decreases in the lateral hypothalamus immediately prior to a migraine headache. ${ }^{31}$ Moreover, resting functional connectivity strength decreases between the lateral hypothalamus and the pain processing pathways (such as the midbrain periaqueductal gray, dorsal pons, rostral ventromedial medulla, and cingulate cortex) before a migraine episode. Abnormal cerebral cortex function can also cause migraine aura symptoms. ${ }^{32}$ Abnormal neural activation within specific consciousness networks, including the prefrontal and posterior parietal cortices, can lead to the alteration of consciousness. Most MBA with DOC patients have a family history of migraine. A variety of mutations in genes (such as CACNA1A, ATP1A2, SCN1A, KCKN18, $P R R T 2$, and $C S N K 1 D)^{33}$ and rare functional gene network abnormalities (such as thyrotropin-releasing hormone receptor and oxytocin receptor signaling pathways, Alzheimer's disease pathway, serotonin receptor pathway, and general heterotrimeric G-protein signaling pathways) are related to migraine. ${ }^{34}$ However, there are no reports of genetic susceptibility in patients with DOC.

The etiology of MBA with DOC is unclear; therefore, patients can only avoid exposure to MBA with DOCinducing factors to reduce the frequency of attacks. The most common predisposing factors ${ }^{17}$ are strong emotional stimulation and sleep disturbance, followed by weather changes, direct sunlight, cold air, high-intensity stress, alcohol consumption, and fatigue.

\section{Clinical Manifestations}

The aura symptoms of MBA are diverse, and when MBA is combined with DOC, the clinical manifestations are more complicated. In this review, we used PubMed, Scopus, Web of Science, and Google Scholar to collect case reports of migraine with DOC from 1961 to 2020 (Table 1). ${ }^{1-15}$ To improve the recall ratio of the literature, we used different search terms besides "migraine with brainstem aura" (including previously used terms: "basilar artery migraine", "basilar migraine", or "basilartype migraine"). Then, we review the relevant literature to further determine whether there was a MBA with
DOC. We found that only five cases (5/27, 18.5\%) meet the current diagnostic criteria according to the third edition of the International Headache Classification (ICHD-3). ${ }^{18}$ This was related to the update of the diagnostic criteria released in 2018. However, this cannot accurately calculate the incidence of MBA with DOC.

\section{Aura Symptoms}

The premonitory symptoms of migraine include, among others, blurred vision, distorted vision, flashing light, nausea, vomiting, photophobia, and sound fear; these can occur several hours to several days before the onset of migraine, ${ }^{35}$ and usually last no more than one hour. ${ }^{36}$ The typical aura symptoms of MBA include vertigo, dysarthria, diplopia, tinnitus, ataxia, and/or DOC, with the most common being diplopia, followed by vertigo, tinnitus, ataxia, and dysarthria. ${ }^{17}$ Notably, motor and retinal symptoms are not considered aura symptoms of MBA according to the ICHD-3 criteria released in $2018 .^{18}$ MBA diagnoses previously reported in the literature may be outdated according to the current diagnostic criteria (Table 1). Most patients have difficulty in accurately describing the duration of aura symptoms due to their discomfort. The duration is mostly estimated by the time periods in medical records. The aura symptoms can be short for some patients (few seconds), while for most of them, the duration ranges from a few minutes to 30 minutes. The longer aura durations reported, ranged from several hours to days (Table 1).

\section{Disorders of Consciousness}

Drowsiness, stupor, and coma are the main terms describing a decreased level of consciousness in MBA with DOC patients, with coma being the most common. The duration of DOC can range from a few seconds ${ }^{14,15}$ to two weeks. ${ }^{7}$ The majority of patients gradually regain consciousness within a few hours; most commonly within half an hour. In coma patients, DOC lasts from a few seconds to a few minutes (Table 1). In general, the duration of DOC in MBA patients is short, and consciousness levels can often return to normal within half an hour.

\section{Headache Characteristics}

Headache often occurs after regaining consciousness or at the same time as DOC. ${ }^{2,13}$ Headaches are frequently severe, accompanied by unbearable, throbbing pain. The most common type of headache is the occipital. About half of the patients have headache symptoms relieved within 


\begin{tabular}{|c|c|c|c|c|c|c|c|c|c|c|c|c|c|c|c|}
\hline$\frac{B}{2} \frac{B}{x}$ & 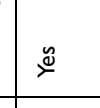 & $\%$ & $\frac{\underline{s}}{1}$ & 8 & 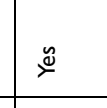 & $\frac{\pi}{z}$ & $\frac{\underline{s}}{2}$ & $\frac{\underline{s}}{z}$ & $\frac{\pi}{z}$ & $\frac{s}{2}$ & 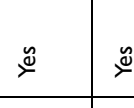 & 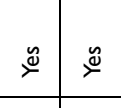 & $\frac{\pi}{2}$ & $\frac{s}{z}$ & 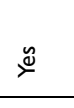 \\
\hline य & 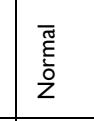 & \begin{tabular}{|l}
$\overline{\bar{s}}$ \\
$\bar{z}$
\end{tabular} & $\frac{\overline{1}}{\frac{1}{2}}$ & \begin{tabular}{|l|}
$\overline{\bar{s}}$ \\
\end{tabular} & \begin{tabular}{|l}
$\frac{\bar{s}}{\underline{\underline{i}}}$ \\
\end{tabular} & 言| & $\frac{\sqrt{\frac{1}{2}}}{2}$ & $\frac{\overline{\bar{s}}}{\underline{\underline{z}}}$ & 譬 & 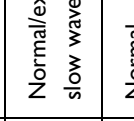 & $\begin{array}{l}\overline{\bar{z}} \\
\overline{2} \\
\overline{2}\end{array}$ & 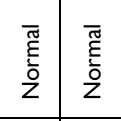 & 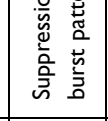 & & 旁 \\
\hline & 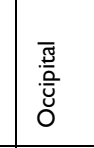 & 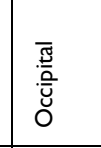 & 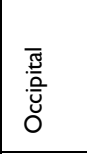 & 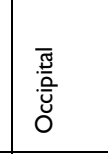 & 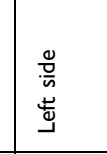 & 荐 & $\frac{\pi}{z}$ & $\frac{x}{z}$ & 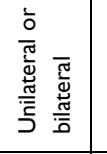 & 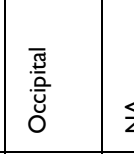 & \begin{tabular}{l|l|l}
$\frac{1}{z}$ & $\frac{1}{2}$ \\
\end{tabular} & $\frac{1}{\Sigma} \frac{\bar{g}}{\bar{z}}$ & 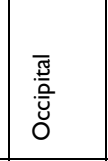 & & 亳 \\
\hline & $\mid$ & $\frac{2}{2}$ & 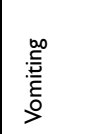 & $\stackrel{2}{2}$ & & & & & 䩧 & 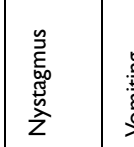 & 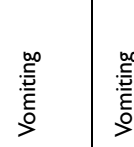 & 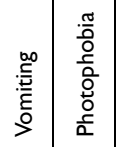 & 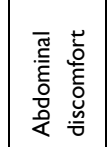 & & \\
\hline 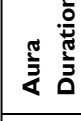 & $\underline{z}$ & 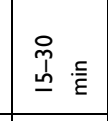 & 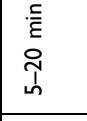 & $\frac{c}{\bar{c}}$ & $\frac{z}{z}$ & $=$ & 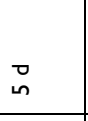 & $\underline{z}$ & $\stackrel{P}{\underline{I}}$ & 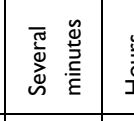 & 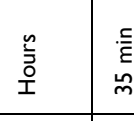 & 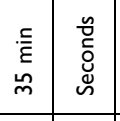 & 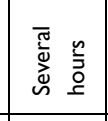 & & $\frac{1}{2}$ \\
\hline & 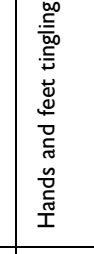 & ஜ & 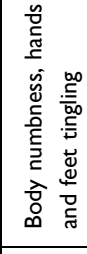 & $z$ & $\stackrel{\circ}{z}$ & $\check{z}$ & 悬 & 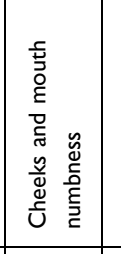 & $\stackrel{2}{2}$ & z & & $\stackrel{2}{2}$ & 尊 & & 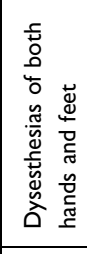 \\
\hline & 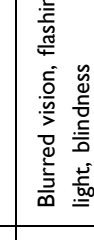 & 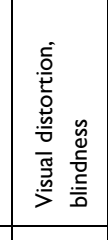 & 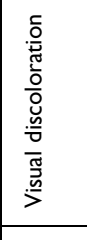 & 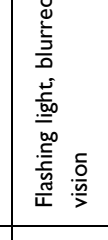 & $\AA$ & 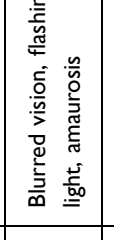 & $\begin{array}{l} \\
\end{array}$ & & & & 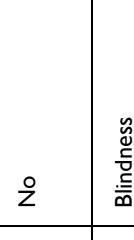 & 亮亮 & z & & z \\
\hline ๘ & $\stackrel{\circ}{z}$ & & 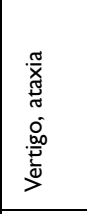 & 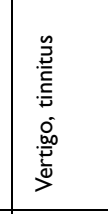 & 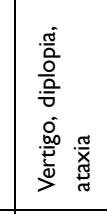 & 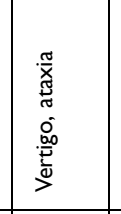 & 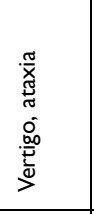 & $\stackrel{\circ}{z}$ & & & & 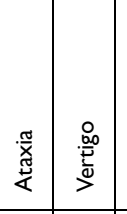 & 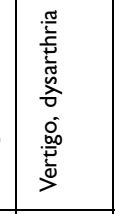 & & \\
\hline & 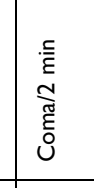 & 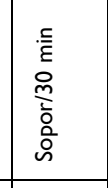 & $\frac{\bar{v}}{\bar{c}}$ & 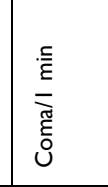 & 密 & $\frac{5}{5}$ & & 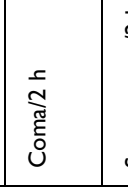 & {$\left[n^{\circ}\right.$} & & 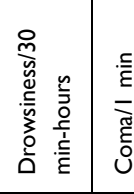 & 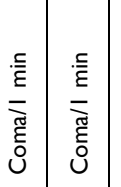 & 喬 & & $\mid$ \\
\hline$\Delta$ & $\frac{ \pm}{ \pm}$ & $\mid \frac{ \pm}{ \pm}$ & $\frac{\approx}{\bar{\Sigma}}$ & $\frac{\frac{2}{\Sigma}}{\underline{4}}$ & 童 & $\frac{\frac{7}{2}}{\bar{z}}$ & $\overline{\underline{\Sigma}}$ & 㱐 & $\frac{2}{\pi}$ & \begin{tabular}{|l|l} 
\pm \\
\multirow{2}{*}{}
\end{tabular} & 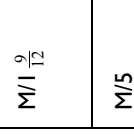 & 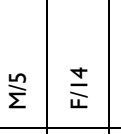 & $\frac{\mu}{2}$ & & 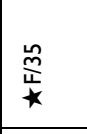 \\
\hline
\end{tabular}




\begin{tabular}{|c|c|c|c|c|c|c|c|c|c|c|c|}
\hline 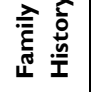 & 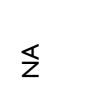 & $\stackrel{\tilde{y}}{\tau}$ & $\mathbb{z}$ & $\stackrel{\tilde{u}}{\tau}$ & $\stackrel{y}{\check{\nu}}$ & $\stackrel{\Xi}{*}$ & $\stackrel{\tilde{u}}{\tau}$ & $\stackrel{\tilde{\nu}}{\check{\nu}}$ & $\S$ & 우 & $\stackrel{0}{z}$ \\
\hline 导 & $\begin{array}{l}0 \\
0 \\
3 \\
3 \\
z \\
0 \\
0\end{array}$ & 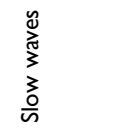 & \begin{tabular}{|l}
$\overline{\widetilde{g}}$ \\
$\overline{\tilde{g}}$ \\
$\mathrm{Q}$
\end{tabular} & $\overleftarrow{z}$ & $\begin{array}{l}\overline{\widetilde{g}} \\
\text { हे } \\
z\end{array}$ & $\begin{array}{l}\overline{\bar{g}} \\
\overline{\underline{\underline{g}}} \\
\mathrm{z}\end{array}$ & $\begin{array}{l}\overline{\widetilde{g}} \\
\bar{E} \\
z \bar{z}\end{array}$ & $\begin{array}{l}0 \\
\sum_{13} \\
0\end{array}$ & $\begin{array}{l}0 \\
\sum_{3}^{0} \\
\infty\end{array}$ & $\begin{array}{l}\overline{\widetilde{c}} \\
\bar{E} \\
\text { ż }\end{array}$ & $\begin{array}{l}\overline{\widetilde{\sigma}} \\
\overline{\tilde{O}} \\
2\end{array}$ \\
\hline 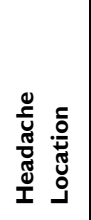 & 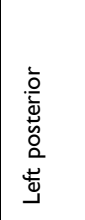 & $\mathbb{z}$ & 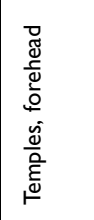 & 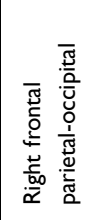 & $\mathbb{Z}$ & 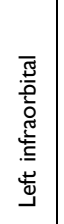 & 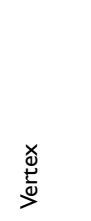 & 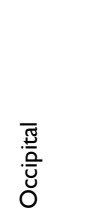 & 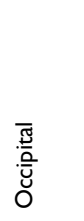 & 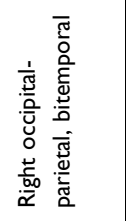 & 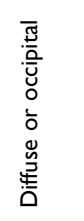 \\
\hline 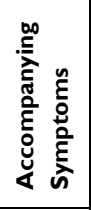 & $\stackrel{\circ}{z}$ & 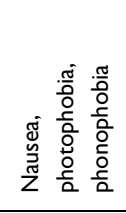 & 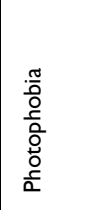 & 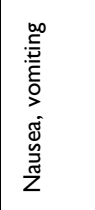 & 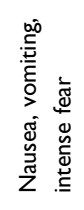 & \begin{tabular}{l|}
$\frac{0}{0}$ \\
$\frac{0}{0}$ \\
$\frac{0}{0}$ \\
$\frac{0}{0}$ \\
$\frac{0}{\alpha}$
\end{tabular} & 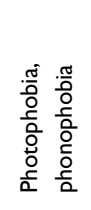 & $\stackrel{0}{z}$ & $\stackrel{0}{z}$ & 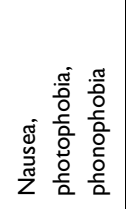 & zo \\
\hline 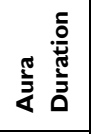 & 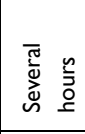 & 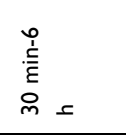 & $\begin{array}{l}\stackrel{c}{\xi} \\
\underline{\underline{O}}\end{array}$ & 도 & 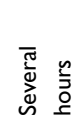 & 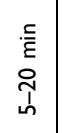 & $\begin{array}{l}\text { 毫 } \\
\text { n }\end{array}$ & $\frac{\mathscr{n}}{\underline{d}} \cdot \frac{c}{\varepsilon}$ & $\begin{array}{l}\frac{c}{\bar{\varepsilon}} \\
\frac{0}{1} \\
\omega^{\prime}\end{array}$ & in & $\begin{array}{l}\frac{c}{\bar{\varepsilon}} \\
\stackrel{\rho}{\rho}\end{array}$ \\
\hline 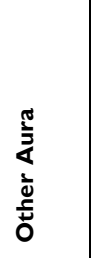 & 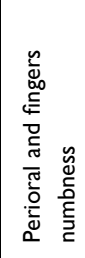 & io & ㅇ & z & 우 & 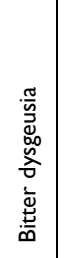 & 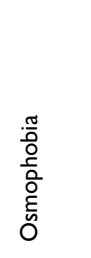 & 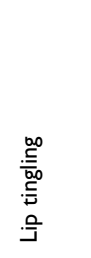 & 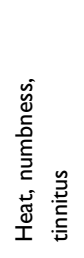 & 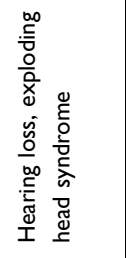 & 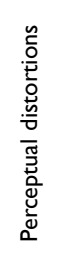 \\
\hline 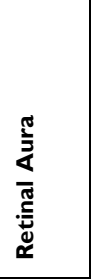 & 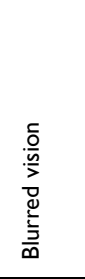 & 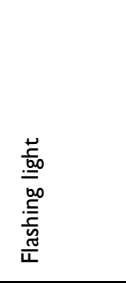 & $\stackrel{0}{z}$ & 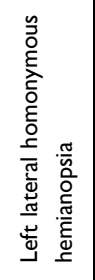 & $\stackrel{\circ}{z}$ & 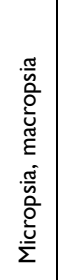 & $\stackrel{0}{z}$ & 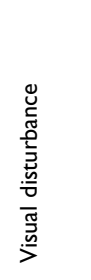 & $\stackrel{0}{z}$ & 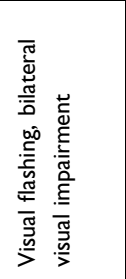 & 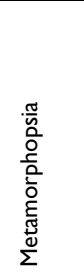 \\
\hline 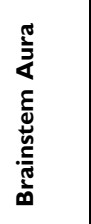 & 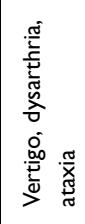 & 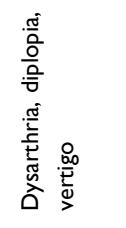 & 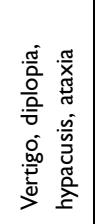 & zo & $\begin{array}{l}\stackrel{\circ}{.0} \\
\stackrel{0}{5} \\
\stackrel{0}{\nu}\end{array}$ & 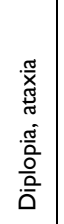 & 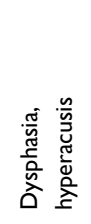 & 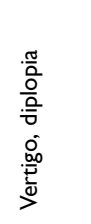 & $\begin{array}{l}\frac{0}{\overline{0}} \\
\frac{0}{\circ} \\
\overline{0}\end{array}$ & 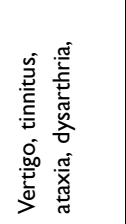 & 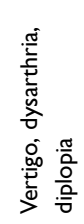 \\
\hline 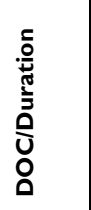 & 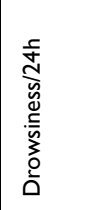 & 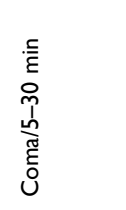 & 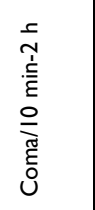 & 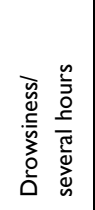 & 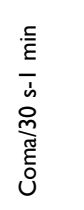 & 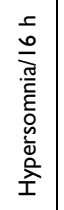 & 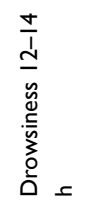 & 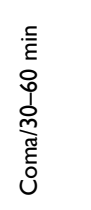 & 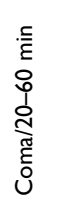 & 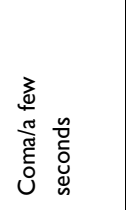 & 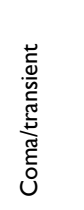 \\
\hline 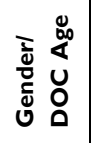 & $\hat{\bar{\Sigma}}$ & $\frac{O}{\Sigma}$ & 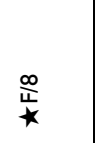 & $\stackrel{0}{\frac{0}{4}}$ & $\sum_{\star}^{\infty}$ & $\bar{\Psi}$ & $\frac{\mathfrak{N}}{\Sigma}$ & $\frac{N}{\Delta)}$ & 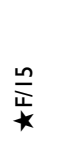 & $\frac{\hat{f}}{\Sigma}$ & $\hat{\bar{\Sigma}}$ \\
\hline 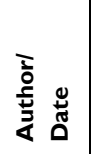 & 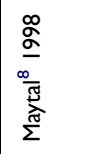 & 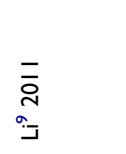 & & 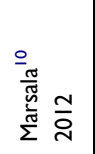 & $\begin{array}{l}\frac{m}{a} \\
\bar{a} \\
\overline{0} \\
\stackrel{0}{0} \\
\underline{\underline{a}}\end{array}$ & 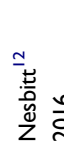 & & 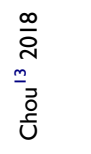 & & 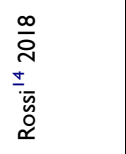 & 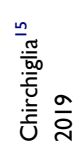 \\
\hline
\end{tabular}


a few hours, while others can relieve headache symptoms by vomiting ${ }^{1,3,4}$ or sleeping..$^{12,13,37}$

\section{Diagnostic Tools}

Routine blood tests and cerebrospinal fluid tests for MBA with DOC patients yield normal results, ${ }^{1,3-15,37-39}$ except for a single case that reported elevated blood ammonia. ${ }^{2}$ Abnormalities in MBA with DOC patients are usually reversible, as indicated by studies employing electroencephalogram (EEG), transcranial Doppler (TCD), magnetic resonance imaging (MRI), single-photon emission computed tomography (SPECT) and neuroelectrophysiological examination. In $30 \%$ of patients, $\mathrm{EEG}^{2,5-9,13,38}$ showed a diffuse slow-wave rhythm during a DOC attack; a result commonly obtained from the occipital lobe, followed by the frontal or bilateral temporal lobe. There are no sharp waves, sharp-slow complex waves, or other epileptic waves. EEG becomes normal after recovery or in between the DOC episodes. TCD and MRI can detect basilar artery spasm, ${ }^{5}$ abnormal signals in the occipital lobe ${ }^{8}$ and brain stem capillary dilation during the attack. ${ }^{37}$ SPECT can detect hypoperfusion of occipital cortex and cerebellar hemisphere. ${ }^{38}$ The above abnormal signs are reversible and are related to aura symptoms and headaches. Moreover, studies have shown abnormal brainstem auditory evoked potentials in MBA patients, ${ }^{6}$ which are mainly manifested as the prolonged latency between the III-IV waves, and this abnormality can be normal with the recovery of the clinical symptoms. A case report of MBA with vertebrobasilar dilatation (VBD), ${ }^{39}$ suggested that VBD may cause chronic pathological changes in the brainstem followed by the corresponding symptoms.

\section{Treatment and Outlook}

The main treatments for MBA with DOC aim to relieve symptoms in the acute phase and prevent the recurrence of an attack. Traditional drugs administered during the acute phase include non-steroidal anti-inflammatory drugs, ${ }^{12}$ ergotamines $^{2}$ and 5-HT receptor agonists. ${ }^{40}$ Administration of 5-HT receptor agonists is currently the most common treatment of acute migraine. The 5-HT 1B/1D receptor agonists (triptans) were used to treat MBA patients. ${ }^{41}$ Triptans can induce vasoconstriction, which leads to cardiocerebrovascular side effects; therefore, their use in MBA treatment has ceased. ${ }^{7,9}$ Although triptans have traditionally been contraindicated in MBA under drug instructions, as the understanding of migraine has evolved substantially, multiple publications have suggested the relative safety of triptans in related patient populations. ${ }^{42}$ Evidence of basilar artery constriction as postulated in MBA is lacking. This prohibition of triptans is being reconsidered in the face of evidence suggesting that their use may be safe. ${ }^{43}$

A recent study found that administration of Lasmiditan does not induce the aforementioned side effects; hence, it can be used to relieve the acute symptoms of MBA. ${ }^{44}$ It is currently the first and only 5-HT1F receptor agonist approved by the Food and Drug Administration. ${ }^{45}$ In addition, analysis of the drug's efficacy found that a dose of $200 \mathrm{mg}$ is more effective than a dose of $100 \mathrm{mg}$. ${ }^{46}$ The prevention of migraine should be combined with non-drug measures ${ }^{47}$ such as avoiding triggers, ${ }^{17}$ controlling weight, ${ }^{48}$ and maintaining a good lifestyle; cognitive behavioral therapy ${ }^{49}$ can also reduce the frequency of migraine attacks. The pharmacological treatments for migraine prevention include $\beta$-receptor antagonists, ${ }^{2}$ calcium channel blockers, ${ }^{8,13,15,37,38}$ anti-epileptics, ${ }^{37,39}$ and tricyclic antidepressants. ${ }^{8,38}$ Recent studies ${ }^{50}$ have found that vitamin B12 and folic acid levels are low in patients with migraine. Thus, vitamin B12 and folic acid supplementation can also play a preventive role in migraine. It was recently found that the calcitonin gene-related peptide (CGRP) is involved in the pathophysiology of migraine. ${ }^{51}$ CGRP monoclonal antibodies and receptor antagonists $^{52}$ are now used as a treatment for the acute phase and the prevention of migraine. ${ }^{53,54}$

The first-generation CGRP receptor antagonists, gepants, were effective for abortive treatment of migraine. ${ }^{55}$ However, they were not fully developed because of hepatotoxicity. ${ }^{56}$ Ubrogepant, also known as MK-1602, is an oral, small-molecule CGRP receptor antagonist for acute migraine treatment. ${ }^{57} \mathrm{~A}$ study found that 2 hours after ubrogepant administration, the patient's headache and aura symptoms were relieved. ${ }^{58}$ Unlike other gepants, ubrogepant has no hepatotoxicity at therapeutic doses. ${ }^{59}$ The most common adverse reactions are nausea, somnolence, and dry mouth. The ACHIEVE II Randomized Clinical Trial study found that both 50-mg and $25-\mathrm{mg}$ doses can significantly relieve migraine. ${ }^{60}$ However, only a $50 \mathrm{mg}$ dose helped to reduce the aura. A Phase III, randomized, 52-week extension trial further confirmed the safety of long-term intermittent use of ubrogepant 50 and $100 \mathrm{mg}$ doses. ${ }^{61}$ Short-term use of ubrogepant was not related to an increased risk for adverse events. $^{62}$ The time course of ubrogepant efficacy was demonstrated out to $48 \mathrm{~h},{ }^{63}$ providing evidence of the long-lasting effect. For the subjective feelings of patients, 
the potential benefits of ubrogepant could improve patientcentered satisfaction. ${ }^{64}$

Rimegepant received FDA approval in February, 2020, for acute migraine treatment. ${ }^{65}$ A randomized, phase III, double-blind, placebo-controlled trial found that $75 \mathrm{mg}$ rimegepant, given via orally disintegrating tablets, were more effective than placebo in the acute treatment of migraine. ${ }^{66}$ The $75 \mathrm{mg}$ dose of rimegepant had good tolerance and safety for acute treatment of migraine. ${ }^{67}$ The patients were free of pain attack and free from the most bothersome symptom compared to placebo treated patients. ${ }^{68}$ For migraine prevention, a recently published Phase 2/3, randomized, double-blind, placebo-controlled trial of oral rimegepant indicated the tolerability was similar to that of placebo, and no unexpected or serious safety issues were noted. ${ }^{69}$

In addition to CGRP receptor antagonists, European headache federation guideline recommended monoclonal antibodies (mAbs) targeting the CGRP receptor (erenumab) and the CGRP (eptinezumab, fremanezumab, and galcanezumab) for migraine prevention. ${ }^{70} \mathrm{~A}$ small sample study found that rimegepant with CGRP mAbs, as preventive treatment, was safe and well tolerated. ${ }^{71}$ This suggests that CGRP mAbs combined receptor antagonists have a promising future in the prevention and treatment of migraine. However, it is still necessary to pay attention to the consequences of long-term CGRP blockade, especially when combined with ischemic diseases and when other anti-migraine drugs are used. ${ }^{72}$

In recent years, there have been some potential biomarkers that can be measured in plasma for migraine patients: (1) the abundance of CD4+ effector memory helper T lymphocytes; ${ }^{73}$ (2) high serum cystatin $\mathrm{C}$ level; $;{ }^{74}$ (3) elevated plasma CGRP level ${ }^{75-77}$ (4) increased Apo E protein levels; ${ }^{78}$ (5) mRNA expression of prostacyclin receptor in peripheral blood lymphocytes; ${ }^{79}$ (6) increased interictal vasoactive intestinal peptide level; ${ }^{80}$ (7) decreased serum S100B level. ${ }^{81}$ Among the abovementioned biomarkers, CGRP seems to be a popular target. However, there were also conflicting studies that found serum CGRP concentration may not be a feasible biomarker for chronic migraine. ${ }^{82}$ Unfortunately, there is no research on the MBA with DOC subtype.

\section{Conclusion}

The basilar artery is less likely to be involved in the onset of MBA and the third edition of the International Headache Classification (ICHD-3) released by IHS has proclaimed that the term "migraine with brainstem aura" will replace the original term "basilar artery migraine" or "basilar-type migraine". ${ }^{18}$ Many MBA patients have multiple typical aura symptoms. ${ }^{32}$ At this time, migraine with typical aura and MBA should be diagnosed simultaneously. Notably, many patients exhibiting motor or retinal symptoms were diagnosed with MBA; however, these symptoms are excluded in the diagnostic criteria for MBA and therefore the diagnosis was outdated according to the ICHD-3 criteria (Table 1).

MBA is a rare disease with complex and diverse aura symptoms. Patients may be referred to the relevant departments when the first symptoms of aura start. Coma is the most common clinical manifestation in MBA with DOC patients. Although the overall duration of the patient's DOC is short and can often return to normal within half an hour, it is still necessary for clinicians to improve their understanding of the disease to avoid misdiagnosis. Although triptans have traditionally been contraindicated in MBA under drug instructions, the evidence of basilar artery constriction as postulated in MBA is lacking. This prohibition of triptans is being reconsidered in the face of evidence suggesting that their use may be safe. Lasmiditan is currently the first and only 5-HT $1 \mathrm{~F}$ receptor agonist approved by the Food and Drug Administration. Calcitonin gene-related peptide receptor antagonists and monoclonal antibody therapies may be the most promising for future consideration.

\section{Funding}

This study was supported by grants from Shanxi Provincial Health and Family Planning Commission (No. 2017033); Doctoral Fund of the First Hospital of Shanxi Medical University (No. YB161706, No. BS03201631); Shanxi Applied Basic Research Program (No. 201801D221426).

\section{Disclosure}

The authors report no conflicts of interest in this work.

\section{References}

1. Bickerstaff E. Impairment of consciousness in migraine. Lancet. 1961;2(7211):1057-1059. doi:10.1016/s0140-6736(61)92538-7

2. Lee C, Lance J. Migraine stupor. Headache. 1977;17(1):32-38. doi:10.1111/j.1526-4610.1977.hed1701032.x

3. Hockaday J. Basilar migraine in childhood. Dev Med Child Neurol. 1979;21(4):455-463. doi:10.1111/j.1469-8749.1979.tb01649.x

4. Smith M, Glass S. An adolescent girl with headache and syncope. $J$ Adolesc Healthc. 1989;10(1):54-56. doi:10.1016/0197-0070(89) 90049-1 
5. Frequin S, Linssen W, Pasman J, Hommes O, Merx H. Recurrent prolonged coma due to basilar artery migraine. A case report. Headache. 1991;31(2):75-81. doi:10.1111/j.1526-4610.1991.hed3102075.x

6. Ganji S, Hellman S, Stagg S, Furlow J. Episodic coma due to acute basilar artery migraine: correlation of EEG and brainstem auditory evoked potential patterns. Clin EEG. 1993;24(1):44-48. doi:10.1177/ 155005949302400110

7. Muellbacher W, Mamoli B. Prolonged impaired consciousness in basilar artery migraine. Headache. 1994;34(5):282-285. doi:10.1111/j.1526-4610.1994.hed3405282.x

8. Maytal J, Libman R, Lustrin E. Basilar artery migraine and reversible imaging abnormalities. AJNR Am J Neuroradiol. 1998;19(6):1116-1119.

9. Li Q, Tan G, Zhou J. Basilar-type migraine with coma: case reports and literature review. Pain Med. 2011;12(4):654-656. doi:10.1111/ j.1526-4637.2011.01080.x

10. Marsala S, Gioulis M. Basilar migraine mistaken for encephalitis. Neurolog Sci. 2012;33(1):213-214. doi:10.1007/s10072-011-0658-5

11. Inoue $\mathrm{Y}$, Yabe $\mathrm{T}$. Lomerizine therapy for the treatment of benign paroxysmal vertigo of childhood transitioning into atypical basilar migraine: a case report. Exp Ther Med. 2013;5(6):1573-1575. doi:10.3892/etm.2013.1035

12. Nesbitt A, Leschziner G. Migraine with brainstem aura presenting as recurrent hypersomnia (Kleine-Levin syndrome). Pract Neurol. 2016;16(5):402-405. doi:10.1136/practneurol-2016-001418

13. Chou I, Lee I, Hong S. Basilar-type migraine: an overlooked cause of loss of consciousness in pediatric emergency rooms. Pediatr Neonatol. 2018;59(3):315-316. doi:10.1016/j.pedneo.2017.08.010

14. Rossi F, Gonzalez E, Rossi E, Tsakadze N. Exploding head syndrome as aura of migraine with brainstem aura: a case report. J Orofac Pain Headache. 2018;32(2):e34-e36. doi:10.11607/ofph.1950

15. Chirchiglia D, Chirchiglia P, Marotta R. A singular association of migraine with brainstem aura and Alice in Wonderland syndrome. Childs Nerv Syst. 2019;35(8):1435-1437. doi:10.1007/s00381-019-04170-8

16. Kirchmann M, Thomsen L, Olesen J. Basilar-type migraine: clinical, epidemiologic, and genetic features. Neurology. 2006;66(6):880-886 doi:10.1212/01.wnl.0000203647.48422.dd

17. Ying G, Fan W, Li N, et al. Clinical characteristics of basilar-type migraine in the neurological clinic of a university hospital. Pain Med. 2014;15(7):1230-1235. doi:10.1111/pme.12402

18. [No authors listed]. Headache Classification Committee of the International Headache Society (IHS) The International Classification of Headache Disorders, 3rd edition. Cephalalgia. 2018;38(1):1-211. doi:10.1177/0333102417738202

19. Chaudhry P, Friedman D. Hiccups as a migraine aura. Cephalalgia. 2015;35(9):831-834. doi:10.1177/0333102414560633

20. Bickerstaff E. Basilar artery migraine. Lancet. 1961;277 (7167):15-17. doi:10.1016/S0140-6736(61)92184-5

21. Kondziella D, Bender A, Diserens K, van Erp W. European academy of neurology guideline on the diagnosis of coma and other disorders of consciousness. Eur J Neurol. 2020;27(5):741-756. doi:10.1111/ ene. 14151

22. Nagata E. Recent advances in the elucidation of migraine pathophysiology. Rinsho Shinkeigaku. 2020;60(1):20-26. doi:10.5692/clinicalneurol.cn-001348

23. Shevel E. The extracranial vascular theory of migraine-a great story confirmed by the facts. Headache. 2011;51(3):409-417. doi:10.1111/ j.1526-4610.2011.01844.x

24. Charles A, Baca S. Cortical spreading depression and migraine. Nat Rev Neurol. 2013;9(11):637-644. doi:10.1038/nrneurol.2013.192

25. Cui Y, Kataoka Y, Watanabe Y. Role of cortical spreading depression in the pathophysiology of migraine. Neurosci Bull. 2014;30 (5):812-822. doi:10.1007/s12264-014-1471-y

26. Ramachandran R. Neurogenic inflammation and its role in migraine. Semin Immunopathol. 2018;40(3):301-314. doi:10.1007/s00281-0180676-y
27. Edvinsson L, Haanes K, Warfvinge K. Does inflammation have a role in migraine? Nat Rev Neurol. 2019;15(8):483-490. doi:10.1038/ s41582-019-0216-y

28. Cavestro C, Ferrero M, Mandrino S, Di Tavi M, Rota E. Novelty in Inflammation and Immunomodulation in Migraine. Curr Pharm Des. 2019;25(27):2919-2936. doi:10.2174/1381612825666190709204107

29. Aguila M, Rebbeck T, Leaver A, et al. The association between clinical characteristics of migraine and brain GABA levels: an Exploratory Study. J Pain. 2016;17(10):1058-1067. doi:10.1016/j.jpain.2016.06.008

30. Requena I, Indakoetxea B, Lema C, Santos B, García-Castiñeira A, Arias M. Coma associated with migraine. Revista Neurologia. 1999;29(11):1048-1051.

31. Meylakh N, Marciszewski K, Di Pietro F, Macefield V, Macey P, Henderson L. Altered regional cerebral blood flow and hypothalamic connectivity immediately prior to a migraine headache. Cephalalgia. 2020;40(5):448-460. doi:10.1177/0333102420911623

32. Demarquay G, Ducros A, Montavont A, Mauguiere F. Migraine with brainstem aura: why not a cortical origin? Cephalalgia. 2018;38 (10):1687-1695. doi:10.1177/0333102417738251

33. Sutherland H, Griffiths L. Genetics of migraine: insights into the molecular basis of migraine disorders. Headache. 2017;57 (4):537-569. doi:10.1111/head.13053

34. Rasmussen A, Kogelman L, Kristensen D, Chalmer M, Olesen J, Hansen T. Functional gene networks reveal distinct mechanisms segregating in migraine families. Brain. 2020;143(10):awaa242. doi:10.1093/brain/awaa242

35. Karsan N, Goadsby P. Biological insights from the premonitory symptoms of migraine. Nat Rev Neurol. 2018;14(12):699-710. doi:10.1038/s41582-018-0098-4

36. Headache Classification Committee of the International Headache Society (IHS). The International Classification of Headache Disorders, 3rd edition (beta version). Cephalalgia. 2013;33(9):629-808. doi:10.1177/ 0333102413485658

37. Beukers R, Roos Y. Pontine capillary telangiectasia as visualized on MR imaging causing a clinical picture resembling basilar-type migraine: a case report. $J$ Neurol. 2009;256(10):1775-1777. doi:10.1007/s00415-009-5204-5

38. Cannas A, Solla P, Mascia M, et al. Medulloblastoma induces unusual headache with clinical picture of basilar-type migraine complicated by ischaemic infarcts. Cephalalgia. 2006;26(10):1238-1241. doi:10.1111/j.1468-2982.2006.01176.x

39. Huang L, Yu C, Wang B, Zhang H, Li L, Wang Y. Vertebrobasilar dolichoectasia causing a presentation resembling basilar-type migraine. Clin Neurol Neurosurg. 2013;115(6):784-786. doi:10.1016/j.clineuro.2012.07.007

40. Huang P, Yang F, Chang C, Yang C. Targeting the 5-HT and 5-HT receptors for acute migraine treatment. Prog Brain Res. 2020;255:99-121. doi:10.1016/bs.pbr.2020.05.010

41. Negro A, Koverech A, Martelletti P. Serotonin receptor agonists in the acute treatment of migraine: a review on their therapeutic potential. J Pain Res. 2018;11:515-526. doi:10.2147/jpr.S132 833

42. Vgontzas A, Burch R. episodic migraine with and without aura: key differences and implications for pathophysiology, management, and assessing risks. Curr Pain Headache Rep. 2018;22(12):78. doi:10.1007/s11916-018-0735-z

43. Mathew PG, Klein BC. Getting to the heart of the matter: migraine, triptans, DHE, ditans, CGRP antibodies, First/Second-Generation gepants, and cardiovascular risk. Headache. 2019;59(8):1421-1426. doi:10.1111/head.13601

44. Curto M, Cipolla F, Cisale G, et al. Profiling lasmiditan as a treatment option for migraine. Expert Opin Pharmacother. 2020;21 (2):147-153. doi:10.1080/14656566.2019.1694004

45. Lamb Y. Lasmiditan: first approval. Drugs. 2019;79(18):1989-1996. doi:10.1007/s40265-019-01225-7 
46. Yang Y, Sun Y, Gao B, Wang Z, Chen Z, Wang Z. Lasmiditan for acute treatment of migraine in adults: a systematic review and meta-analysis of Randomized Controlled Trials. CNS Drugs. 2020;34(10):1015-1024. doi:10.1007/s40263-020-00753-1

47. Hamann T, Kamm K, Kropp P, Rimmele F, Jürgens T. Migraine prophylaxis-all just antibodies?: medical and nonmedical migraine preventive treatment in times of CGRP antibodies. Schmerz. 2020;34 (6):476-485. doi:10.1007/s00482-020-00500-x

48. Kristoffersen E, Børte S, Hagen K, Zwart J, Winsvold B. Migraine, obesity and body fat distribution - a population-based study. $J$ headache Pain. 2020;21(1):97. doi:10.1186/s10194-020-01 163 -w

49. Oskoui M, Pringsheim T, Billinghurst L, et al. Practice guideline update summary: pharmacologic treatment for pediatric migraine prevention: report of the Guideline Development, Dissemination, and Implementation Subcommittee of the American Academy of Neurology and the American Headache Society. Neurology. 2019;93 (11):500-509. doi:10.1212/wnl.0000000000008105

50. Aydin H, Bucak I, Geyik M. Vitamin B12 and folic acid levels in pediatric migraine patients. Acta Neurol Belg. 2020. doi:10.1007/ s13760-020-01491-3

51. Meßlinger K. Pathophysiological role of calcitonin gene-related peptide (CGRP) in migraine and cluster headache. Schmerz. 2020;34 (2):181-187. doi:10.1007/s00482-020-00448-y

52. Goadsby P, Dodick D, Ailani J, et al. Safety, tolerability, and efficacy of orally administered atogepant for the prevention of episodic migraine in adults: a double-blind, randomised phase $2 b / 3$ trial. Lancet Neurol. 2020;19(9):727-737. doi:10.1016/s1474-4422(20) 30234-9

53. Charles A, Pozo-Rosich P. Targeting calcitonin gene-related peptide: a new era in migraine therapy. Lancet. 2019;394(10210):1765-1774. doi:10.1016/s0140-6736(19)32504-8

54. Ferrari M, Diener H, Ning X, et al. Fremanezumab versus placebo for migraine prevention in patients with documented failure to up to four migraine preventive medication classes (FOCUS): a randomised, double-blind, placebo-controlled, Phase 3b trial. Lancet. 2019;394 (10203):1030-1040. doi:10.1016/s0140-6736(19)31946-4

55. Hong P, Tan T, Liu Y, Xiao J. Gepants for abortive treatment of migraine: a network meta-analysis. Brain Behav. 2020;10(8):e01701. doi:10.1002/brb3.1701

56. Moreno-Ajona D, Perez-Rodriguez A, Goadsby PJ. Gepants, calcitonin-gene-related peptide receptor antagonists: what could be their role in migraine treatment? Curr Opin Neurol. 2020;33 (3):309-315. doi:10.1097/WCO.0000000000000806

57. Curto M, Capi M, Cipolla F, Cisale GY, Martelletti P, Lionetto L. Ubrogepant for the treatment of migraine. Expert Opin Pharmacother. 2020;21(7):755-759. doi:10.1080/14656566.202 0.1721462

58. Dodick DW, Lipton RB, Ailani J, et al. Ubrogepant for the treatment of migraine. N Engl J Med. 2019;381(23):2230-2241. doi:10.1056/ NEJMoa1813049

59. Dhir A. Ubrogepant to treat migraine. Drugs Today (Barc). 2020;56 (7):459-467. doi:10.1358/dot.2020.56.7.3157311

60. Lipton RB, Dodick DW, Ailani J, et al. Effect of ubrogepant vs placebo on pain and the most bothersome associated symptom in the acute treatment of migraine: the ACHIEVE II Randomized Clinical Trial. JAMA. 2019;322(19):1887-1898. doi:10.1001/ jama.2019.16711

61. Ailani J, Lipton RB, Hutchinson S, et al. Long-term safety evaluation of ubrogepant for the acute treatment of migraine: phase 3, randomized, 52-Week Extension Trial. Headache. 2020;60(1):141-152. doi:10.1111/head.13682

62. Yang Y, Chen M, Sun Y, Gao B, Chen Z, Wang Z. Safety and efficacy of ubrogepant for the acute treatment of episodic migraine: a meta-analysis of Randomized Clinical Trials. CNS Drugs. 2020;34 (5):463-471. doi:10.1007/s40263-020-00715-7
63. Goadsby PJ, Blumenfeld AM, Lipton RB, et al. Time course of efficacy of ubrogepant for the acute treatment of migraine: clinical implications. Cephalalgia. 2020:333102420970523. doi:10.1177/ 0333102420970523

64. Dodick DW, Lipton RB, Ailani J, et al. Ubrogepant, an acute treatment for migraine, improved patient-reported functional disability and satisfaction in 2 single-attack phase 3 Randomized Trials, ACHIEVE I and II. Headache. 2020;60(4):686-700. doi:10.1111/head.13766

65. Negro A, Martelletti P. Rimegepant for the treatment of migraine. Drugs Today (Barc). 2020;56(12):769-780. doi:10.1358/dot.2020.5 6.12 .3211624

66. Croop R, Goadsby PJ, Stock DA, et al. Efficacy, safety, and tolerability of rimegepant orally disintegrating tablet for the acute treatment of migraine: a randomised, phase 3, double-blind, placebo-controlled trial. Lancet. 2019;394(10200):737-745. doi:10.1016/S0140-6736(19)31606-X

67. Gao B, Yang Y, Wang Z, et al. Efficacy and safety of rimegepant for the acute treatment of migraine: evidence from Randomized Controlled Trials. Front Pharmacol. 2019;10:1577. doi:10.3389/fphar.2019.01577

68. Lipton RB, Croop R, Stock EG, et al. Rimegepant, an oral calcitonin gene-related peptide receptor antagonist, for migraine. $N \mathrm{Engl} \mathrm{J} \mathrm{Med}$. 2019;381(2):142-149. doi:10.1056/NEJMoa1811090

69. Croop R, Lipton RB, Kudrow D, et al. Oral rimegepant for preventive treatment of migraine: a phase $2 / 3$, randomised, double-blind, placebo-controlled trial. Lancet. 2021;397(10268):51-60. doi:10.1016/S0140-6736(20)32544-7

70. Sacco S, Bendtsen L, Ashina M, et al. European headache federation guideline on the use of monoclonal antibodies acting on the calcitonin gene related peptide or its receptor for migraine prevention. J Headache Pain. 2019;20(1):6. doi:10.1186/s10194-018-0955-y

71. Berman G, Croop R, Kudrow D, et al. Safety of rimegepant, an oral CGRP receptor antagonist, plus CGRP monoclonal antibodies for migraine. Headache. 2020;60(8):1734-1742. doi:10.1111/head.13930

72. de Vries T, Al-Hassany L, MaassenVanDenBrink A. Evaluating rimegepant for the treatment of migraine. Expert Opin Pharmacother. 2021;1-7. doi:10.1080/14656566.2021.1895749

73. Pavelek Z, Soucek O, Krejsek J, et al. The role of the immune system and the biomarker CD3 + CD4 + CD45RA-CD62L- in the pathophysiology of migraine. Sci Rep. 2020;10(1):12277. doi:10.1038/s41598020-69285-4

74. Akda GT, Uca AU. Cystatin C as a potential biomarker to evaluate migraine. Arq Neuropsiquiatr. 2020;78(6):337-341. doi:10.1590/ 0004-282X20200005

75. Cernuda-Morollon E, Larrosa D, Ramon C, Vega J, MartinezCamblor P, Pascual J. Interictal increase of CGRP levels in peripheral blood as a biomarker for chronic migraine. Neurology. 2013;81 (14):1191-1196. doi:10.1212/WNL.0b013e3182a6cb72

76. Ramon C, Cernuda-Morollon E, Pascual J. Calcitonin gene-related peptide in peripheral blood as a biomarker for migraine. Curr Opin Neurol. 2017;30(3):281-286. doi:10.1097/WCO.0000000000000440

77. Fan PC, Kuo PH, Lee MT, Chang SH, Chiou LC. Plasma calcitonin gene-related peptide: a potential biomarker for diagnosis and therapeutic responses in pediatric migraine. Front Neurol. 2019;10:10. doi:10.3389/fneur.2019.00010

78. Yuasa N, Nagata E, Fujii N, Ito M, Tsukamoto H, Takizawa S. Serum apolipoprotein E may be a novel biomarker of migraine. PLoS One. 2018;13(1):e0190620. doi:10.1371/journal.pone.0190620

79. Kheirollahi M, Kazemi M, Amini G, et al. Expression of prostaglandin I2 (prostacyclin) receptor in blood of migraine patients: a potential biomarker. Adv Biomed Res. 2015;4(1):121. doi:10.4103/ 2277-9175.158030

80. Cernuda-Morollon E, Martinez-Camblor P, Alvarez R, Larrosa D, Ramon C, Pascual J. Increased VIP levels in peripheral blood outside migraine attacks as a potential biomarker of cranial parasympathetic activation in chronic migraine. Cephalalgia. 2015;35(4):310-316. doi: $10.1177 / 0333102414535111$ 
81. Celikbilek A, Sabah S, Tanik N, Ak H, Atalay T, Yilmaz N. Is serum S100B protein an useful biomarker in migraine? Neurol Sci. 2014;35 (8):1197-1201. doi:10.1007/s10072-014-1679-7
82. Lee MJ, Lee SY, Cho S, Kang ES, Chung CS. Feasibility of serum CGRP measurement as a biomarker of chronic migraine: a critical reappraisal. $J$ Headache Pain. 2018;19(1):53. doi:10.1186/s10194-018-0883-x

\section{Publish your work in this journal}

The Journal of Pain Research is an international, peer reviewed, open access, online journal that welcomes laboratory and clinical findings in the fields of pain research and the prevention and management of pain. Original research, reviews, symposium reports, hypothesis formation and commentaries are all considered for publication. The manuscript

Submit your manuscript here: https://www.dovepress.com/journal-of-pain-research-journal management system is completely online and includes a very quick and fair peer-review system, which is all easy to use. Visit http:// www.dovepress.com/testimonials.php to read real quotes from published authors. 